\title{
Correction to: Excavating Al: the politics of images in machine learning training sets
}

\author{
Kate Crawford ${ }^{1} \cdot$ Trevor Paglen $^{2}$
}

Accepted: 4 October 2021 / Published online: 23 November 2021

(c) Springer-Verlag London Ltd., part of Springer Nature 2021

\section{Correction to: AI \& SOCIETY https://doi.org/10.1007/s00146-021-01162-8}

The Editor-in-Chief has removed Fig. 1 due to copyright and consent concerns, along with the related references, and Fig. 2a has been updated. A sentence in Sect. 3 "Anatomy of a training set" explaining the authors' understanding of the intended purpose of the JAFFE dataset (Lyons and Akamatsu 1998) has also been removed.

Additionally, the Authors would like to provide the following clarifications:

- Figure 2 gives examples of images from the ImageNet database, which was first introduced in the cited article (Deng et al. 2009)

- On page 6 , the following sentence incorrectly references a figure: "A photo shopped picture shows a smiling Barack Obama wearing a Nazi uniform, his arm raised and holding a Nazi flag. It is labelled "Bolshevik (Fig. 2)."' The citation to Fig. 2 should have been placed in the following paragraph when referencing the labelled image of Sigourney Weaver

- The images in Fig. 3 were reproduced from the "Aligned\&Cropped Faces" dataset of UTKFace (UTKFace-Aicip 2019)

- The full caption for Fig. 4 is: "Fig. 4 image from IBM's Diversity in Faces paper, image credit M. Merler et al. (Merler et al. 2019).

The original article can be found online at https://doi.org/10.1007/ s00146-021-01162-8.

Kate Crawford

Kate.crawford@usc.edu

Trevor Paglen

trevor@paglen.com

1 University of Southern California, Annenberg School, Microsoft Research New York, New York, USA

2 The University of Georgia, Athens, Greece

\section{References}

Deng J, Dong W, Socher R, Li L, Li K, Fei-Fei L (2009) Imagenet: a large-scale hierarchical image database. In: IEEE conference on computer vision and pattern recognition, pp 248-255. https://doi. org/10.1109/CVPR.2009.5206848

Lyons M, Akamatsu S (1998) Coding facial expressions with gabor wavelets. In: Proceedings, third IEEE international conference on automatic face and gesture recognition, 14-16 April 1998, Nara, Japan. IEEE Computer Society, pp 200-205. https://doi.org/10. 1109/AFGR.1998.670949

Merler M, Ratha N, Feris RS, Smith JR (2019) Diversity in faces. ArXiv 4:1901-10436. arXiv:1901.10436

UTKFace-Aicip. http://aicip.eecs.utk.edu/wiki/UTKFace. Accessed 28 Aug 2019

Publisher's Note Springer Nature remains neutral with regard to jurisdictional claims in published maps and institutional affiliations. 